\title{
SUPPRESSION OF PROINFLAMMATORY CYTOKINE PRODUCTION BY SUBEROYLANILIDE HYDROXAMIC ACID AN INHIBITOR OF HISTONE DEACETYLASES
}

\author{
Z. Dobreva*, S. Stanilova \\ Department of Molecular Biology, Immunology and Medical Genetics, Medical Faculty, \\ Trakia University, Stara Zagora, Bulgaria
}

\begin{abstract}
ABSRACT
Chromatin remodeling by acetylation and deacetylation of histones play an essential role in regulation of the gene expression. The opposing activity of two type enzymes controls the acetylation degree of the chromatin structure. Histone acetylation by acetyltransferases leads to relaxed chromatin structure and this process supports the transcription. Deacetylation by histone deacetylases (HDACs) compact the chromatin structure and favors gene silence. Small molecule inhibitors of HDACs are a new class drugs used in clinical trials for the treatment of various malignancies. Emerging evidence suggest that HDAC inhibitors may have also anti-inflammatory properties, although the molecular mechanisms remain poorly defined. Our study investigates the effect of the HDACs inhibitor Suberoylanilide Hydroxamic Acid (SAHA) on Th1 and Th17 polarizing cytokines IL-12p40 and IL-23. For this purpose, human peripheral blood mononuclear cells (PBMC) were stimulated with LPS and C3bgp with or without SAHA. Cytokine production was determined in culture supernatants at 6 and $24 \mathrm{~h}$, by ELISA. IL-12p 40 and IL-23 synthesis was measured in stimulated PBMC at 6 and increased at $24 \mathrm{~h}$. Early at $6 \mathrm{~h}$, we detected significantly decreased IL-12p40 and IL-23 production in stimulated PBMC treated with SAHA and this tendency was retained at $24 \mathrm{~h}$. In conclusion, our results demonstrated that the inhibition of HDACs by SAHA leads to suppression of IL-12p40 and IL-23 regardless of stimuli used. The downregulating effect of SAHA on the proinflammatory cytokine production may be beneficial during treatment of chronic inflammatory diseases which progression is mediated by Th17 immune response.
\end{abstract}

Key words: HDAC, IL-10, IL-12p40, IL-23, SAHA

\section{INTRODUCTION}

During immune response naïve Th cells differentiate into different subsets, with different cytokine production profiles and effector functions. Until recently, T cells were divided into Th1 or Th2 cells, depending on the cytokines they produce (1). Th1 cells produce large quantities of IFN- $\gamma$ and mediate cellular immunity while Th2 cells, which are involved in humoral immunity, primarily produce IL-4, IL-5, and IL-13 (2). IL-12, produced by antigen presenting cells (APC), promotes the differentiation of naive CD4+ Th cells into IFN- $\gamma$-producing Th 1 cells. IL-12 is a heterodimeric cytokine composed of two disulfide-linked proteins - p40 and p35. The p40 subunit also binds to other protein p19 to

\footnotetext{
* Correspondence to: Assoc. Prof. Z.G. Dobreva, PhD, Department of Molecular Biology, Immunology and Medical Genetics, Medical Faculty, Trakia University, Bulgaria, E-mail: zdobreva@mf.uni-sz.bg
}

form IL-23 - cytokine that similarly to IL-12 is produced by APC. IL-23 is a main cytokine responsible for the expansion of the third, currently discovered subset of the effector Th lymphocytes Th17, characterized by the production of IL-17. The significance of Th17 cells in the immune response is the defense against certain types of pathogens, particularly when endangering epithelial surfaces (3). However, Th17 cells are potent inducers of tissue inflammation and have been associated with the pathogenesis of many experimental autoimmune diseases and inflammatory conditions (1). Of this point of view elevated levels of IL-23 may have a prognostic significance for the development of pathogenic Th17 immune response.

Chromatin remodeling by acetylation and deacetylation of histones play an essential role in the regulation of the gene expression (4). The opposing activity of two type enzymes controls the acetylation degree of the 
chromatin structure. Histone acetylation by acetyltransferases leads to relaxed chromatin structure and is generally assumed that this process supports the transcription. Deacetylation by histone deacetylases (HDACs) compact the chromatin structure and thus favoring gene silence (5). HDACs are grouped into four distinct classes. Class I HDACs which includes HDAC1, 2, 3 and 8 are generally localized to the nucleus. Class I HDACs have been most widely studied in their classical role as histone modifiers and transcriptional repressors. Class II HDACs HDAC4, 5, 6, 7, 9 and 10, can shuttle between the nucleus and cytoplasm and have been subdivided into class IIa and IIb. Nuclear export prevents class IIa HDACs from acting as transcriptional repressors, thus resulting in inducible gene expression. In some cases, class IIa HDACs can act as transcriptional activators or control the gene expression by recruiting other proteins (6). HDAC11 is the sole member of the class IV. Class III HDACs or SIRT1-7 require the cofactor nicotinamide adenine dinucleotide for its activity and act via a distinct mechanisms than class I, II and IV HDACs (7).

Histone deacetylase inhibitors (HDI) are small organic molecules which prevent the action of HDACs and thus maintain the acetylation degree of histones. Via effects on chromatin structure and transcription factor binding, they modulate the expression of $-2-10 \%$ of cellular genes (8). Although histone acetylation is generally associated with transcriptional activation, HDI can either increase or decrease expression of specific genes. HDI are of prime interest in cancer research because they induce apoptosis and cell cycle arrest in malignant cells and are anti-angiogenic (9, 10). Suberoylanilide Hydroxamic Acid (SAHA) is the HDI targeted to the class I and class II HDAC enzymes (7). HDAC inhibitor SAHA was FDA-approved in 2006 for the treatment of cutaneous T-cell lymphoma as vorinostat (11). There were evidence that SAHA selectively up-regulated the expression of many genes in tumor cells, triggering cellcycle arrest and apoptosis (4). SAHA caused specific modifications in the pattern of acetylation and methylation of lysines in histones $\mathrm{H} 3$ and $\mathrm{H} 4$ associated with the proximal promoter of the p21 gene. SAHA also induced a marked decrease in HDAC1, Myc, and recruitment of RNA polymerase II in the protein complex associated with this promoter region (12). A growing body of evidence suggest that HDI including SAHA in addition to their anticancer properties also can influence immune response via suppression of proinflammatory cytokines such as TNF$\alpha$ (10), IL- 12p40 (13), IL-1 and IFN- $\gamma$ (14) at protein and mRNA level. The role of HDACs in the regulation of cytokine synthesis from activated immune cells, however, is not exactly elucidated.

In the current study we evaluated the in vitro effects of SAHA on the lipopolysaccharide (LPS) - and C3binding glycoprotein (C3bgp) induced proinflammatory Th1 and Th17 driving cytokines IL-12p40 and IL-23 produced by human peripheral blood mononuclear cells (PBMC).

\section{MATERIAL AND METHODS \\ Reagents}

Lipopolysaccharide (LPS) from Escherichia coli serotype 026:B6, Histopaque-1077, HDAC inhibitor Suberoylanilide Hydroxamic Acid (SAHA), and all culture reagents were obtained from Sigma-Aldrich. Polystyrene materials were manufactured by Corning Inc. IL-12p40 and IL-23 production was measured using commercially available kits purchased from Invitrogen, Life Technologies. C3 binding glycoprotein (C3bgp) was isolated as described previously (15).

\section{PBMC isolation}

With the approval of the local ethics board, blood samples were taken from 12 healthy donors. The informing consent was obtained from each participant. The peripheral venous blood $(10 \mathrm{ml})$ was collected in sterile tubes with ethylenediamine tetraacetic acid (EDTA). Peripheral blood mononuclear cells (PBMC) were isolated by Histopaque-1077 density gradient centrifugation. The interface containing PBMC was harvested and washed twice with cold RPMI-1640 medium.

\section{Cell cultures and stimulation}

PBMC $\left(1 \times 10^{6}\right.$ cells $\left./ \mathrm{ml}\right)$ cultures were carried out in RPMI-1640 supplemented with: $10 \%$ FBS, $100 \mathrm{U} / \mathrm{ml}$ penicillin, $100 \mu \mathrm{g} / \mathrm{ml}$ gentamicin and $0.3 \mathrm{mg} / \mathrm{ml} \mathrm{L}$-glutamine. The cells were stimulated with: $30 \mu \mathrm{g} / \mathrm{ml} \mathrm{C} 3 \mathrm{bgp}$ or $1 \mu \mathrm{g} / \mathrm{ml}$ LPS. PBMC cultures were incubated at $37^{\circ} \mathrm{C}$ for $6 \mathrm{~h}$ or $24 \mathrm{~h}$. After incubation, the cultures were centrifuged of $1800 \mathrm{rpm}$ for 10 min. Supernatants was separated and stored at $70^{\circ} \mathrm{C}$ until use.

\section{Inhibition of histone deacetylases}

For the inhibition of HDACs we used the HDAC inhibitor SAHA. $5 \mu \mathrm{m}$ SAHA was added $1 \mathrm{~h}$ before stimulation to PBMC cultures. SAHA was dissolved in $100 \%$ dimethylsulphoxide (DMSO) and the final 
concentration of DMSO in cultures was $0.1 \%$. To avoid the influence of DMSO on the cytokine synthesis, nonstimulated cell cultures (controls) with $0.1 \%$ DMSO were seeded.

\section{Cytokine determination}

The quantity determination of IL-12p40 and IL-23 was performed by ELISA in culture supernatants according to the manufacturer's protocols. Color reaction developed was measured as OD units at $450 \mathrm{~nm}$. The concentration of each cytokine was determined by using standard curve constructed with kit's standards and was expressed in $\mathrm{pg} / \mathrm{ml}$. The minimum detectable concentration of the IL$12 \mathrm{p} 40$ ELISA kit was less than $2 \mathrm{pg} / \mathrm{ml}$ and of IL-23 $<23 \mathrm{pg} / \mathrm{ml}$.

\section{Statistical analysis}

The data was expressed as means and standard error of the mean. Student's t-test was used to determine the statistical differences between mean values. Differences were considered significant when the $\mathrm{P}$ value was less than 0.05 .

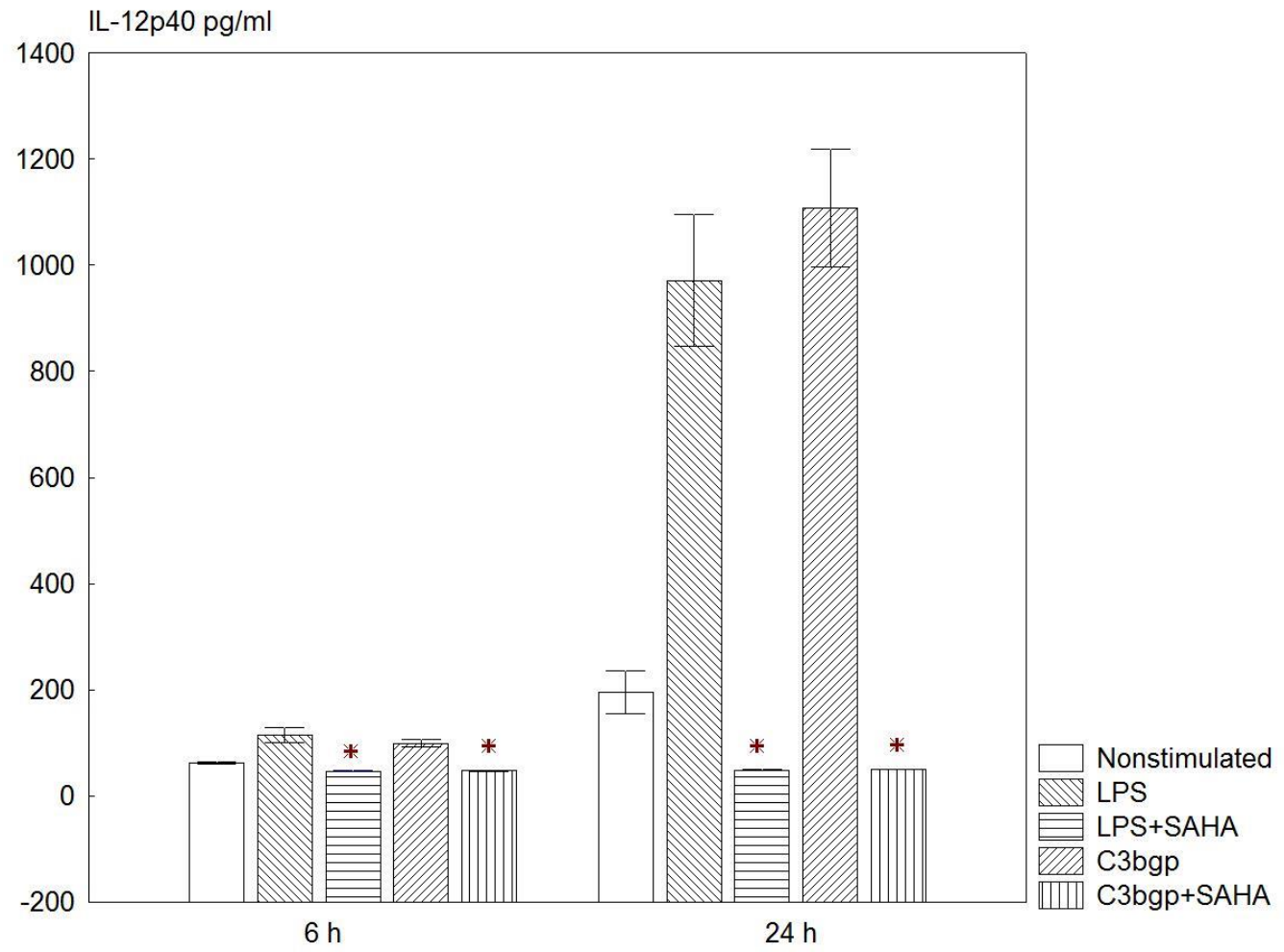

Figure 1. IL-12p40 production from stimulated with LPS and C3bgp PBMC after inhibition of HDAC by $5 \mu$ g SAHA. *Significantly less $(\mathrm{p}<0.05)$ than LPS and C3bgp stimulated cultures at 6 and $24 \mathrm{~h}$.

The inhibition of HDAC with SAHA decreased the production of IL-23

LPS and C3bgp evoked significantly higher IL-23 production at $6 \mathrm{~h}$ (Figure 2) in comparison with control cultures $(\mathrm{p}=0.000007$ for LPS, $\mathrm{p}=0.0002$ for C3bgp). IL-23 protein level at $24 \mathrm{~h}$ in stimulated group was greatly enhanced versus unstimulated control ( $\mathrm{p}=0.011$ for LPS and $\mathrm{p}=0.004$ for C3bgp) and versus

\section{RESULTS}

The inhibition of HDAC with SAHA decreased the production of IL-12p40

In response to the stimuli used PBMC produced significantly enhanced IL-12p40 at 6 $\mathrm{h}$, compared to the nonstimulated control ( $\mathrm{p}=0.013$ for LPS, $\mathrm{p}=0.00011$ for C3bgp) (Figure 1). This tendency was clearly demonstrated at $24 \mathrm{~h}$ when LPS and C3bgp induced the higher protein quantity in comparison with both control group $(\mathrm{p}=0.0001$ for LPS and $\mathrm{p}=0.000004$ for C3bgp) and the same cultures at $6 \mathrm{~h}(\mathrm{p}=0.000001$ for LPS and $\mathrm{p}=0.000000$ for C3bgp).

The inhibition of HDAC with SAHA resulted in significantly diminished IL-12p40 synthesis at $6 \mathrm{~h}(\mathrm{p}=0.00007$ for LPS vs. LPS+SAHA, $\mathrm{p}=0.000001$ for C3bgp vs, C3bgp+SAHA). The inhibitory effect of SAHA was highly expressed at $24 \mathrm{~h}$, with statistical significance $\mathrm{p}=0.000000$ for both LPS vs. LPS + SAHA and C3bgp vs. C3bgp + SAHA. the same cultures at $6 \mathrm{~h}(\mathrm{p}=0.002$ for LPS and $\mathrm{p}=0.0005$ for C $3 \mathrm{bgp}$ ).

We observed significantly decreased IL-23 production in stimulated cultures treated with SAHA at $6 \mathrm{~h}(\mathrm{p}=0.0001$ for LPS vs. LPS+SAHA, $\mathrm{p}=0.0006$ for C3bgp vs. C3bgp+SAHA). Again at $24 \mathrm{~h}$ the inhibition of HDAC with SAHA resulted 
in significant reduction of IL-23 protein synthesis $\quad(p=0.0001$ for LPS vs.

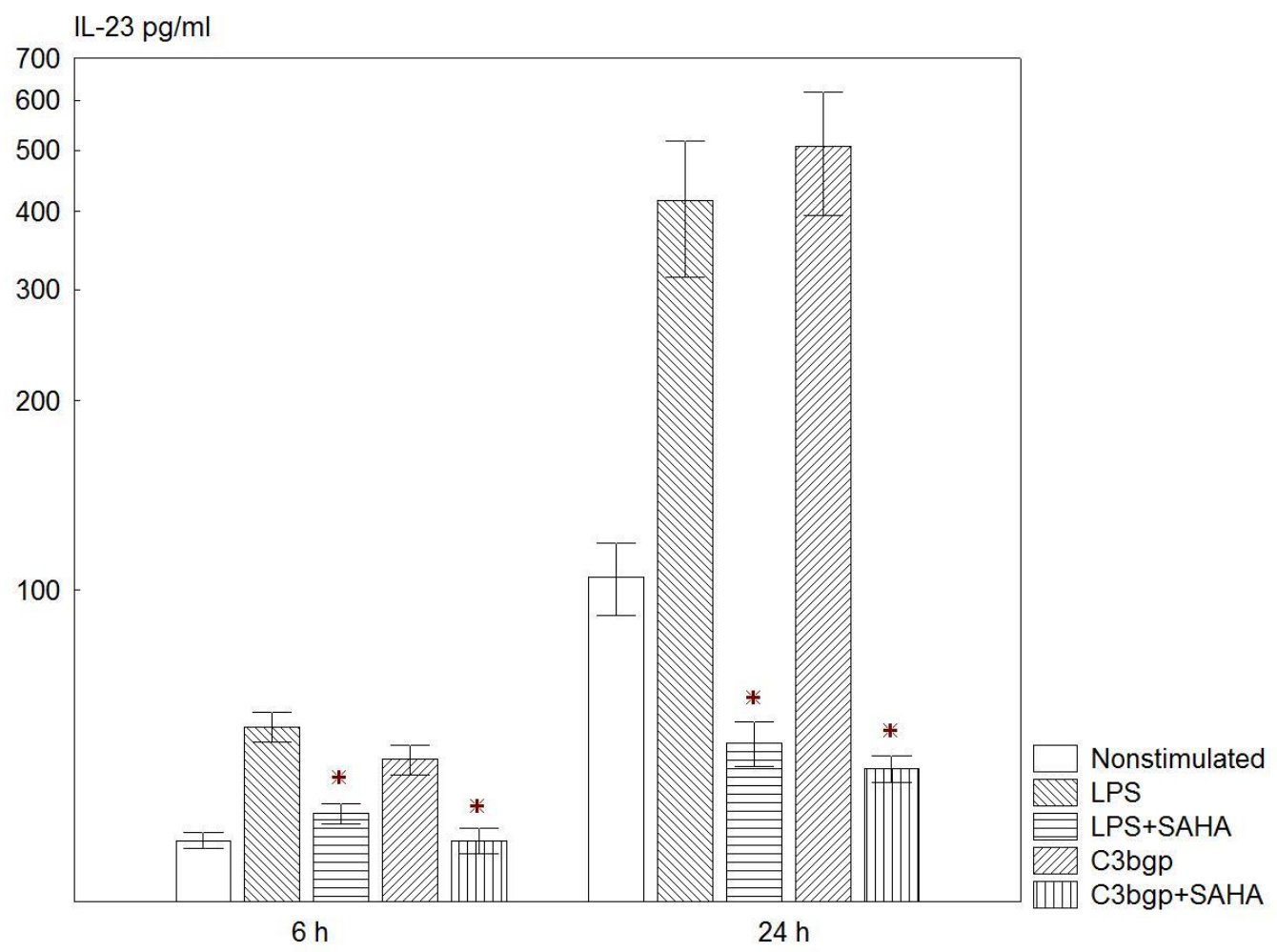

Figure 2. IL-23 production from stimulated with LPS and C3bgp PBMC after inhibition of HDAC by 5 $\mu$ g SAHA. *Significantly less $(\mathrm{p}<0.05)$ than LPS and C3bgp stimulated cultures at 6 and $24 \mathrm{~h}$.

\section{DISCUSSION}

HDAC inhibitors are a new class drugs with promising anticancer effects. By inhibition of HDACs activity these compounds induce cellcycle arrest and apoptosis $(9,10)$. In addition, there were increasing evidence suggesting that HDAC inhibitors, influenced proinflammatory cytokine synthesis $(10,14)$. Nevertheless, the molecular mechanisms of this action remain poorly defined.

Our study demonstrated that the inhibition of HDAC by $5 \mu \mathrm{m}$ SAHA strongly reduced proinflammatory IL-12p40 and IL-23 secretion from stimulated PBMC. Similar results regarding IL-12p40 and IL-23 at mRNA and protein level were reported by other authors ( 3 , $5,7,13,16)$. Regardless of the stimuli used, the inhibitory effect of SAHA was powerful and was occurred rapidly - reduced quantity of IL-12p40 and IL-23 was detected in cultures treated with SAHA, early at $6 \mathrm{~h}$. Bearing in mind the inhibitory effect of SAHA on class I and II HDACs we assumed that this effect may occurred because of two reasons - i) lack of deacetylation of histones associated with $I L$ $12 B$ and $I L-23 A$ genes; ii) lack of deacetylation of transcription factors and proteins involved in transduction pathways which are activated by LPS and C3bgp. Actually, Butler et al. reported an incremental accumulation of acetylated histones $\mathrm{H} 3$ and $\mathrm{H} 4$ in LNCaP cells cultured for $3 \mathrm{~h}$ with $0.5-5 \mu \mathrm{m}$ SAHA (17). Although histone acetylation is associated with the expression of the target genes histone deacetylase inhibitors decrease the expression of proinflammatory cytokines by a still unknown mechanism. For example, Bode et al. 2007, reported that, HDAC inhibitors increased the acetylation status of IL-12p40 locus. Nevertheless, IL-12p40 remodeling, binding of transcription factors Rel-A и IRF1 to the IL-12p40 promoter and transcriptional activation is abrogated (13).

It is well know, that LPS-stimulated proinflammatory cytokine production was mediated by TLR4 and NF-kB transduction pathway. Our previously published data suggest that C3bgp mediated its activity through mitogen activated protein kinases (MAPK) JNK and p38 (18). Because of the inhibitory effect of SAHA on the LPS- and C3bgp - stimulated cytokine production we conclude that HDAC positively regulates both $\mathrm{NF}-\mathrm{kB}$ and mitogen activated protein kinase JNK and p38 transduction pathways. In addition to their histone targets HDACs have a number of non-histone protein targets including transcription factors, transcription regulators, signal transduction mediators and other proteins (12). In fact the work of Choo et 
DOBREVA Z., et al.

al., demonstrated that SAHA suppress p38 MAPK in synovial fibroblastic E11 cells (19). Concerning NF-kB activity, it has been reported that HDAC-mediated deacetylation alters the transcriptional activity of nuclear transcription factors, including NF-kB (20). In addition, in their review article Shakespear et al. comment that TLR inducible expression of several interferon regulatory factor (IRF) transcription factor family target genes, including $I L 12 b$, is dependent upon HDAC activity in macrophages and DCs (6). Furthermore, Bode et al. showed that HDAC inhibitors SAHA and TSA reduced recruitment of IRF1 to DNAse hypersensitive site in the $I L 12 b$ promoter in mouse BMDCs (13). Therefore, the data cited above support our assumption that general mechanisms by which SAHA reduced proinflammatory IL-12p40 and IL-23 expression include both histone modification and recruitment of transcription factors.

Currently, is widely accepted that IL-23 drive the expansion of Th17 cells, inducers of pathogenic autoimmune and inflammatory response. Therefore, the levels of IL-23 can serve as a prognostic value for the development of harmful immune response. IL23 elevated levels of mRNA of two subunits of IL-23 p19 and p40 were reported by Li et al., in human microglia in patients with multiple sclerosis (21). In their investigation Kim et al., showed that the levels of IL-23p19 in the sera and synovial fluid were much higher in patients with rheumatoid arthritis than in osteoarthritis patients or healthy controls (22). Increased expression of IL-23 p19 and p40 in skin lesion of patients with psoriasis vulgaris was reported by Lee et al. (23). In Crohn disease Fuss et al., found also increased IL-12 and IL-23 levels and used monoclonal anti-IL12 p40 antibody for the treatment of patients (24). In addition, IL-23 is upregulated in many human carcinomas and has been suggested to provide a tumor promoting environment. For example a strong upregulation of IL-23 expression has been observed by Stanilov et al., in patients with advanced colorectal cancer at mRNA and protein levels $(25,26)$.

In conclusion, current study examines suppressive activities of SAHA on Th1 and Th17 driving IL-12p40 and IL-23 production. Regardless of the stimuli used SAHA reduced IL-12p40 and IL-23 production from peripheral blood mononuclear cells. Our findings confirmed the conclusion that downregulating effects of SAHA on the IL12 p40 and IL-23 production may be beneficial during treatment of Th17 driving inflammatory and autoimmune diseases.

\section{ACKNOWLEDGMENTS}

This work was supported by Grant No: 3/2013 from the Fund for Scientific and Mobile project from Faculty of Medicine at the Trakia University, Stara Zagora, Bulgaria.

\section{REFERENCES}

1. Korn, T., Bettelli, E., Oukka, M., Kuchroo, V.K., IL-17 and Th17 Cells. Annu Rev Immunol, 27:485-517, 2009.

2. Iwakura, Y. and Ishigame, H., The IL23/IL-17 axis in inflammation. $J$ Clin Invest, 116: 1218-1222, 2006.

3. Bosisio, D., Vulcano, M., Del Prete, A., Sironi, M., Salvi, V., Salogni, L., Riboldi, E., Leoni, F., Dinarello, C.A., Girolomoni, G., and Sozzani, S., Blocking TH17polarizing cytokines by histone deacetylase inhibitors in vitro and in vivo. $J$ Leukoc Biol, 84: 1540 - 1548, 2008.

4. Li, N., Zhao, D., Kirschbaum, M., Zhang, C., Lin, C.L., Todorov, I., Kandeel, F., Forman, S., and Zeng, D., HDAC inhibitor reduces cytokine storm and facilitates induction of chimerism that reverses lupus in anti-CD3 conditioning regimen. PNAS, 105: 4796-4801, 2008.

5. Roger, T., Lugrin, J., Le Roi, D., Goy, G., Mombelli, M., Koessier, T., Ding, X.C., Chanson, A.L., Reymond, M.K., Miconnet, I., Schrenzel, J., Francois, P., and Calandra, T., Histone deacetylase inhibitors impair innate immune response to Toll-like receptor agonist and to infection. Blood, 117: 1205-1217, 2011.

6. Shakespear, M.R., Halili, M.A., Irvine, K.M., Fairlie, D.P., and Sweet, M.J., Histone deacetylases as regulators of inflammation and immunity. Trends in Immunology, 32: 335-343, 2011.

7. Halili, M.A., Andrews, M.R., Labzin, L.I., Schroder, K., Matthias, G., Cao, C., Lovelace, E., Reid, R.C., Le, G.T., Hume, D., Irvine, K.M., Matthias, P., Fairlie, D.P., and Sweet, M.J., Differential effects of selective HDAC inhibitors on macrophage inflammatory responses to the Toll-like receptor 4 agonist LPS. J Leuk Biol, 87:1$11,2010$.

8. Tiffon, C.E., Adams, J.E., Fits, van der L., Wen, S., Townsend, P.A., Ganesan, A., Hodges, E., Vermeer, M.H., and Packham, G., The histone deacetylase inhibitors vorinostat and romidepsin downmodulate IL-10 expression in cutaneous T-cell lymphoma cells. $B r J$ Pharmacol, 162: 1590-1602, 2011. 
9. Bolden, J.E., Peart, M.J., Johnstone, R.W., Anticancer activities of histone deacetylase inhibitors. Nat Rev Drug Discov, 5: 769784, 2006.

10.Wang, Z., Chen C., Finger, S.N., Kwajah, S.M.M., Jung, M., Schwarz, H., Swanson, N., Lareu, R.R., and Raghunath, M., Suberoylanilide hydroxamic acid: a potential epigenetic therapeutic agent for lung fibrosis? Eur Respir J, 34: 145-155, 2009.

11.Ververis, K., Hiong, A., Karagiannis, T.C., Licciardi, P.V., Histone deacetylase inhibitors (HDACIs): multitargeted anticancer agents. Biologics: Targets and Therapy 7: 47-60, 2013.

12.Xu, W.S., Parmigiani, R.B., and Marks, P.A., Histone deacetylase inhibitors: molecular mechanisms of action. Oncogene, 26: 5541-5552, 2007.

13.Bode, K.A., Schroder, K., Hume, D.A., Ravasi, T., Heeg, K., Sweet, M.J., and Dalpke, H.A., Histone deacetylase inhibitors decrease Toll-like receptormediated activation of proinflammatory gene expression by impairing transcription factor recruitment. Immunology, 122: 596606, 2007.

14.Leoni, F., Zaliani A., Bertolini, G., Porro, G., Pagani ,P., Pozzi ,P., Dona, G., Fossati, G., Sozzani, S., Azam, T., Bufler, P., Fantuzzi, G., Goncharov, I., Kim, S.H., Pomerantz, B.J., Reznikov, L.L., Siegmund, B., Dinarello, C.A., and Mascagni, P., The antitumor histone deacetylase inhibitor suberoylanilide hydroxamic acid exhibits antiinflammatory properties via suppression of cytokines. PNAS, 99: 2995-3000, 2002.

15.Zhelev, Z, Stanilova, S, Carpenter, B., Isolation, partial characterization and complement inhibiting activity of a new glycoprotein from Cuscuta europea. Biochem Biophys Res Commun, 202:18694, 1994.

16.Ge, Z., Da, Y.,Xue, Z., Zhang, K., Zhuang, H., Peng, M., Li, Y., Li, W., Simard, A., Hao, J., Yao, Z., Zhang, R,. Vorinostat, a histone deacetylase inhibitor, suppresses dendritic cell function and ameliorates experimental autoimmune encephalomyelitis. Exp Neurol, 241: 56-66, 2013.

17.Butler, L.M., Agus, D.B., Scher, H.I.,, Higgins, B., Rose, A., Cordon-Cardo, C., Thaler, H.T., Rifkind, R.A., Marks, P.A., and Richon, V.M., Suberoylanilide hydroxamic acid, an inhibitor of histone deacetylase, suppresses the growth of prostate cancer cells in vitro and in vivo. Cancer Research, 60: 5165 - 5170, 2000.

18.Dobreva, Z.G., Stanilova, S.A., The immunomodulatory activity of $\mathrm{C} 3$ binding glycoprotein (C3bgp) is mediated by the complement receptor type III and mitogenactivated protein kinase signal transduction pathways. Immunopharmacol \& Immunotoxicol, 29: 549-562, 2007.

19.Choo, Q-Y., Ho, P.C., Tanaka, Y., and Lin, H.S., The histone deacetylase inhibitors MS-275 and SAHA suppress the p38 mitogen-activated protein kinase signaling pathway and chemotaxis in rheumatoid arthritic synovial fibroblastic E11 cells. Molecules, 18: 14085-14095, 2013.

20.Kim, H. J., Bae, S.C., Histone deacetylase inhibitors: molecular mechanisms of action and clinical trials as anti-cancer drugs. Am J Transl Res, 3:166-179, 2011.

21.Li, Y., Chu, N., Hu, A., Gran, B., Rostami, A., Zhang, G. X., Increased IL-23p19 expression in multiple sclerosis lesions and its induction in microglia. Brain, 130: 490501, 2007.

22.Kim, H. R., Cho, M. L., Kim, K. W., Juhn, J. Y., Hwang, S. Y., Yoon, C. H., Park, S. H., Lee, S. H., Kim, H. Y., Up-regulation of IL-23p19 expression in rheumatoid arthritis synovial fibroblasts by IL-17 through PI3kinase-, NF-B- and p38 MAPK-dependent signaling pathways. Rheumatology (Oxford), 46, 57-64, 2007.

23.Lee, E., Trepicchio, W. L., Oestreicher, J. L., Pittman, D., Wang, F., Chamian, F., Dhodapkar, M., Krueger, J. G., Increased expression of interleukin 23 p19 and p40 in lesional skin of patients with psoriasis vulgaris. J Exp Med, 199, 125-130, 2004.

24.Fuss, I. J., Becker, C., Yang, Z., Groden, C., Hornung, R. L., Heller, F., Neurath, M. F., Strober, W., Mannon, P. J., Both IL12 p70 and IL-23 are synthesized during active Crohn's disease and are downregulated by treatment with anti-IL-12 p40 monoclonal antibody. Inflamm Bowel Dis, 12, 9-15, 2006

25.Stanilov, N., Miteva, L., Deliysky, T., Jovchev, J., and Stanilova, S., Advanced colorectal cancer is associated with enhanced IL-23 and IL-10 serum levels. Labmedicine, 41: 159 -163, 2010.

26.Stanilov, N., Miteva, L., Mintchev, N., Stanilova, S., High expression of Foxp3, IL-23p19 and survivin mRNA in colorectal carcinoma. Int J Colorectal Dis, 24:151157, 2009. 